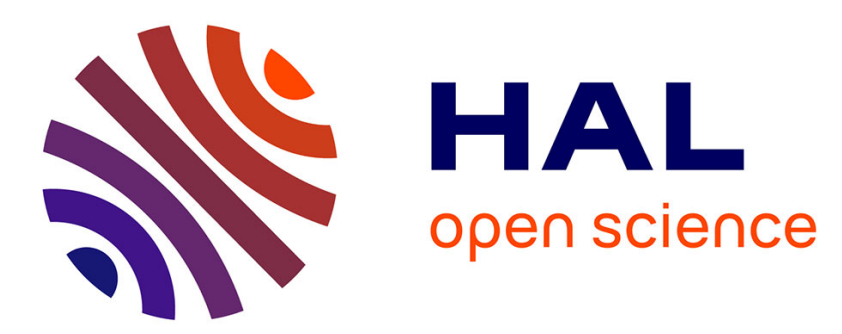

\title{
Behaviour comparison between mechanical epicyclic gears and magnetic gears
}

Melaine Desvaux, B. Multon, Hamid Ben Ahmed, Stéphane Sire

\section{To cite this version:}

Melaine Desvaux, B. Multon, Hamid Ben Ahmed, Stéphane Sire. Behaviour comparison between mechanical epicyclic gears and magnetic gears. Multibody Mechatronic Systems, 54, pp.401-410, 2017, Mechanisms and Machine Science, 10.1007/978-3-319-67567-1_38 . hal-01629812

\section{HAL Id: hal-01629812 \\ https://hal.science/hal-01629812}

Submitted on 6 Nov 2017

HAL is a multi-disciplinary open access archive for the deposit and dissemination of scientific research documents, whether they are published or not. The documents may come from teaching and research institutions in France or abroad, or from public or private research centers.
L'archive ouverte pluridisciplinaire HAL, est destinée au dépôt et à la diffusion de documents scientifiques de niveau recherche, publiés ou non, émanant des établissements d'enseignement et de recherche français ou étrangers, des laboratoires publics ou privés. 


\title{
Behaviour comparison between mechanical epicyclic gears and magnetic gears
}

\author{
M. Desvaux ${ }^{1}$, B. Multon ${ }^{1}$, H. Ben Ahmed ${ }^{1}$ and S. Sire ${ }^{2}$ \\ ${ }^{1}$ SATIE Laboratory, ENS Rennes, Université Bretagne Loire, CNRS, France, e- \\ mail:melaine.desvaux@ens-rennes.fr, bernard.multon@ens-rennes.fr, \\ hamid.benahmed@ens-rennes.fr \\ ${ }^{2}$ Université de Bretagne Occidentale, FRE CNRS 3744, IRDL, France, e-mail: \\ stephane.sire@univ-brest.fr
}

\begin{abstract}
This article deals with the magnetic gears with modulating ring and mechanical epicyclic gears and their operation. A power transmission analysis is done and permits to obtain an analogy of the kinematics between the two gear systems. Moreover, a transposition of the mechanical epicyclic gear arrangements to a magnetic gear arrangements can be made. Finally, a load repartition evolution description is proposed to complete the comparison between magnetic gears with modulating ring and mechanical epicyclic gears.
\end{abstract}

Key words: Arrangements, Gear ratio, Load Repartition, Magnetic gear, Mechanical epicyclic gear.

\section{Introduction}

In many mechatronic drive chains, such as wind turbine drives [1]-[2], mechanical gearbox failure and maintenance increase operating costs, particularly in offshore locations. To improve drive train reliability, one potentially attractive solution consists of replacing the mechanical gearboxes by a magnetic gear [3]. The most studied topology of magnetic gears has been proposed by Martin [4] and was the subject of different behaviour studies proposed by Atallah [3].
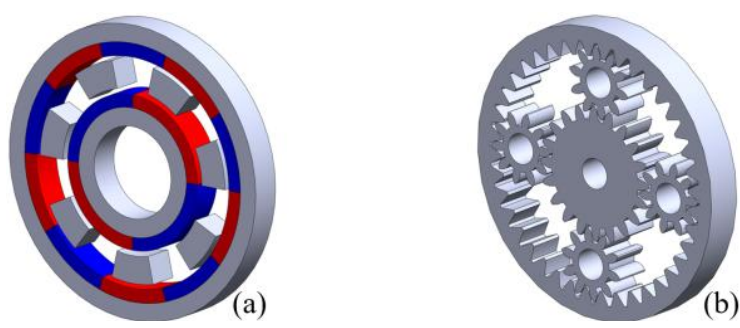

Fig. 1 (a) Magnetic gear with modulating ring [3] and (b) Mechanical epicyclic gear 
The magnetic gear shown in Fig.1a includes an internal ring of permanent magnets, an external ring of permanent magnets and a ring of ferromagnetic pole pieces between the permanent magnets rings.

Different studies show that in wind turbine context, this topology of magnetic gear potentially offers high performance with a higher torque density and a higher reliability than mechanical gearboxes [5][6]. It becomes even more attractive for high-torque applications, like a high-power wind turbine (on the order of several MN.m and several MW) [7].

The behaviour of the magnetic gear with modulating ring [3] can be compared with mechanical epicyclic gear [5] shown in Fig. 1b. Mechanical epicyclic gear is composed of a planetary carrier with its satellites, an internal ring and an external ring. Different topologies of mechanical epicyclic gear have been studied since many years [8] and four epicyclic gear simple arrangements are commonly presented as shown in Fig. 2. In wind turbine, the most attractive arrangement is the epicyclic gear with a single range of satellites (Fig. 2a) since the fact to have only one range of satellites increase the power density of the system.

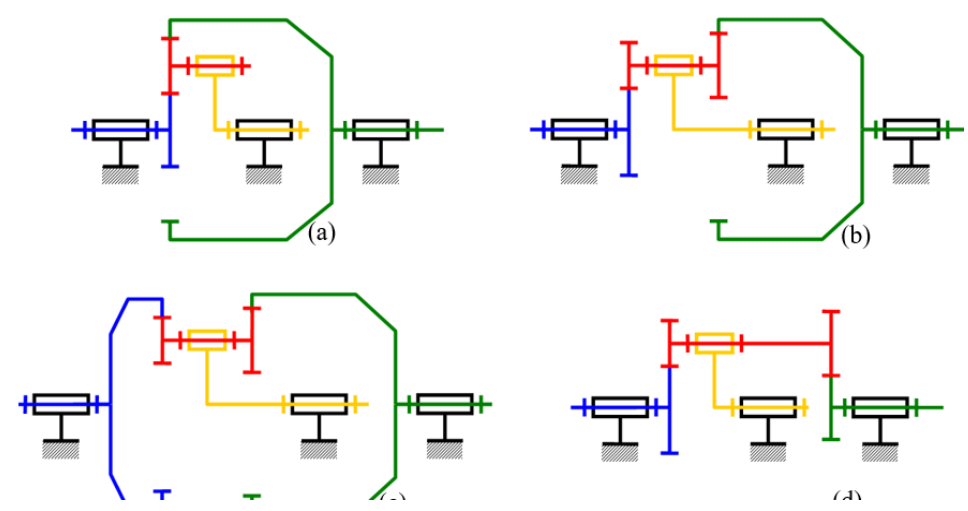

Fig. 2 Schematic epicyclic gear arrangements with (a) an internal ring, an external ring and a planetary carrier with its satellites, (b) an internal ring, an external ring and a planetary carrier with two ranges of satellites, (c) two external rings and a planetary carrier with two ranges of satellites and (d) two internal rings and a planetary carrier with two ranges of satellites.

In this article, the major contribution is the comparison between the four epicyclic gear arrangements and magnetic gear with modulating ring arrangements. It will includes an arrangement, a kinematic (with a study of the gear ratio) and a load repartition approaches. 


\section{Mechanical epicyclic gear behaviour}

To explain the power transmission performed by the mechanical epicyclic gear and compare it with the power transmission of the magnetic gear with modulating ring, the arrangement shown in Fig.1a is studied. Moreover, it is considered that the input of the system is the ring with the high teeth number (which will be called the input ring) and the output of the system is the ring with the low teeth number (which will be called the output ring) as shown in Fig. 3.

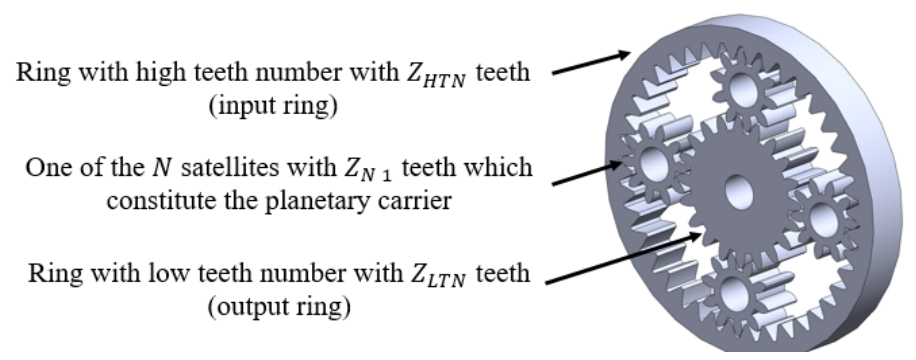

Fig. 3 Rings definition of the mechanical epicyclic gear

\subsection{General principle}

All arrangements of mechanical epicyclic gears are composed of three rings:

- A ring with $Z_{L T N}$ of teeth (with low teeth number) and a module $m_{L T N}$,

- A ring with $Z_{H T N}$ of teeth (with high teeth number) and a module $m_{H T N}$,

- A planetary carrier with one or two ranges of satellites composed of $N$ satellites with $Z_{N i}$ teeth and a module $m_{N}$ for the range $i$ (an example is given in Fig. 3 with: $Z_{L T N}=20, Z_{H T N}=40, Z_{N 1}=Z_{N 2}=10$ and $N=4$ ).

\subsection{Power transmission}

The contact between input ring teeth and the planetary teeth generates friction and then a complex mechanical stress state on the contact point of the $N$ satellites Eq. (1). In the case of a straight toothed gear, only the $\sigma_{r \theta}$ stress component generates tangential loads. For the $N$ satellites, these loads $F_{\text {tang }}^{n}(t)$ defined in Eq. (2) are calculated from the integration of the stress component $\sigma_{r \theta}$ on the contact area $S(t)$. In the same time, the $N$ satellites are in contact with the output ring. From the mechanical equilibrium, satellites transmit directly the tangential loads to the output ring. The mechanical torque on the output ring is then given by Eq. (3). 


$$
\begin{gathered}
\overline{\overline{\sigma_{\text {mech }}}}(M, t)=\left(\begin{array}{ccc}
\sigma_{r r}(t) & \sigma_{r \theta}(t) & \sigma_{r z}(t) \\
\sigma_{r \theta}(t) & \sigma_{\theta \theta}(t) & \sigma_{\theta z}(t) \\
\sigma_{r z}(t) & \sigma_{\theta z}(t) & \sigma_{z z}(t)
\end{array}\right)_{\left(\overrightarrow{e_{r}}, \overrightarrow{\theta_{\theta}}, \overrightarrow{e_{z}}\right)} \\
F_{\text {tang }}^{n}(t)=\int_{S(t)} \sigma_{r \theta}(t) \cdot d S(t) \\
T_{L T N}(t)=R . \sum_{n=1}^{N} F_{\text {tang }}^{n}(t)
\end{gathered}
$$

The mechanical power transmission can be performed if the average value of the mechanical torque is different to zero when the different rings are in rotation. This condition can be made by imposing a single module $m$ defined in Eq. (4) for the different rings and by imposing the Eq. (5) between the teeth number for the different rings (in the general case).

$$
\begin{gathered}
m=m_{L T N}=m_{H T N}=m_{N 1}=m_{N 2} \\
Z_{L T N}+Z_{N 1}=Z_{H T N}+Z_{N 2}
\end{gathered}
$$

With these equations, it is possible to define the ratio $\lambda_{\text {mech }}$ defined in Eq. (6) where $n_{\text {mech }}$ corresponds to the number of rings external to the planetary carrier and the Willis relation for a mechanical epicyclic gear is given in Eq. (7). Then the gear ratio $G_{m e c h}$ depends on the fixed ring as shown in Eq. (8) [9].

$$
\begin{gathered}
\lambda_{\text {mech }}=\frac{\omega_{L T N / 0}-\omega_{P C / 0}}{\omega_{H T N / 0}-\omega_{P C / 0}}=\frac{Z_{H P N} \cdot Z_{N 1}}{Z_{N 2} \cdot Z_{L P N}}(-1)^{n_{m e c h}} \\
\omega_{L T N / 0}-\lambda_{m e c h} \cdot \omega_{H T N / 0}+\left(\lambda_{m e c h}-1\right) \cdot \omega_{P C / 0}=0 \\
\left\{\begin{array}{c}
\omega_{L T N / 0}=0 \rightarrow G_{m e c h}=\frac{\omega_{H T N / 0}}{\omega_{P C / 0}}=\frac{\left(\lambda_{\text {mech }}-1\right)}{\lambda_{m e c h}} \\
\omega_{P C / 0}=0 \rightarrow G_{m e c h}=\frac{\omega_{L T N / 0}}{\omega_{H T N / 0}}=\lambda_{m e c h} \\
\omega_{H T N / 0}=0 \rightarrow G_{m e c h}=\frac{\omega_{L T N / 0}}{\omega_{P C / 0}}=-\left(\lambda_{\text {mech }}-1\right)
\end{array}\right.
\end{gathered}
$$

\section{Magnetic gear with modulating ring behaviour}

To explain the power transmission performed by the magnetic gear [3], it is considered that the input of the system is the low speed permanent magnet ring with the high pole number (which will be called the input permanent magnets ring) and the output of the system is the high speed permanent magnet ring with the low pole number (which will be called the output ring) as shown in Fig. 4. 


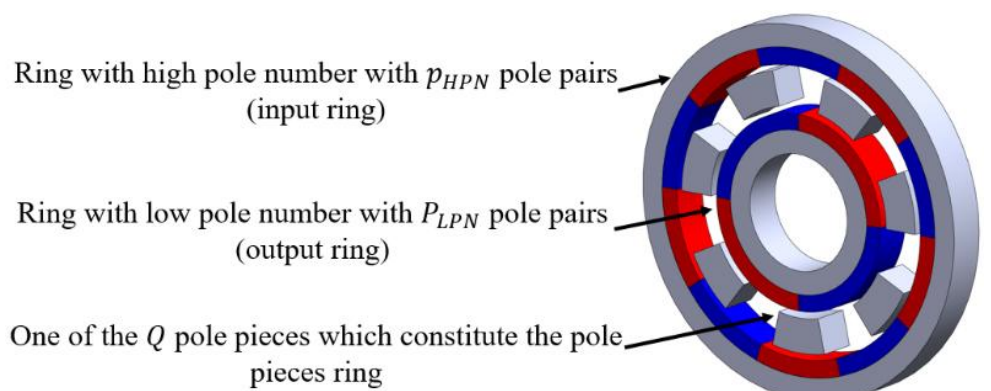

Fig. 4 Definition of the different parts of the studied magnetic gear arrangement

\subsection{General principle}

The general principle of the different magnetic gear with modulating ring arrangements can be described from the magnetic gear [3] which corresponds to the magnetic gear arrangement presented in Fig.4. All arrangements of magnetic gear with modulating ring are composed of three rings:

- A ring with $p_{L P N}$ low pole number of pole pairs of permanent magnets and a ferromagnetic yoke,

- A ring with $p_{H P N}$ high pole number of pole pairs of permanent magnets and a ferromagnetic yoke,

- A ring with $\mathrm{Q}$ ferromagnetic pole pieces (an example is given in Fig. 4 with low pole numbers, to improve readability: $p_{L P N}=2, p_{H P N}=5$ and $Q=7$ ).

Contrary to the mechanical epicyclic gear, pole pieces do not rotate like the satellites on the planetary carrier.

\subsection{Power transmission}

The input permanent magnets ring generates in air gaps a wave of magnetomotive force and then an induction $\overrightarrow{B_{H P N}}$ defined in Eq. (9) in the neighbouring air gap (to simplify the demonstration, only the fundamental wave of the three rings is taken into account). Ferromagnetic pole pieces module the permeance to obtain in the air gap close to the output permanent magnet ring the induction from the input permanent magnet ring $\overrightarrow{B_{m H P N}}$ defined in Eq. (10). There is also in this airgap the induction from the output permanent magnets ring $\overrightarrow{B_{L P N}}$ as shown in Eq. (11). Both inductions produce then a global induction $\overrightarrow{B_{t o t}}$ defined in Eq. (12) close to the output permanent magnets ring.

$$
\overrightarrow{B_{H P N}}(t)=\left(B_{r}^{H P N} \cdot \overrightarrow{e_{r}}+B_{\theta}^{H P N} \cdot \overrightarrow{e_{\theta}}\right) \cos \left(p_{H P N} \cdot \omega_{H P N} \cdot t\right)
$$




$$
\begin{gathered}
\overrightarrow{B_{m H P N}}(t)=\left(B_{m r}^{H P N} \cdot \overrightarrow{e_{r}}+B_{m \theta}^{H P N} \cdot \overrightarrow{e_{\theta}}\right) \cos \left(\left(p_{H P N}-Q\right) \cdot \omega_{H P N} \cdot t\right) \\
\overrightarrow{B_{L P N}}(t)=B_{r}^{L P N}\left(B_{r}^{L P N} \cdot \overrightarrow{e_{r}}+B_{\theta}^{L P N} \cdot \overrightarrow{e_{\theta}}\right) \cos \left(p_{L P N} \cdot \omega_{L P N} \cdot t\right) \\
\overrightarrow{B_{t o t}}(t)=\overrightarrow{B_{m H P N}}(t)+\overrightarrow{B_{L P N}}(t)=B_{r}^{t o t}(t) \cdot \overrightarrow{e_{r}}+B_{\theta}^{t o t}(t) \cdot \overrightarrow{e_{\theta}}
\end{gathered}
$$

The global induction $\overrightarrow{B_{t o t}}$ will generate a mechanical stress state which corresponds to the 2-D Maxwell tensor $\overline{\overline{\sigma_{\text {magn }}}}$ defined in Eq. (13). The instantaneous magnetic torque on the output permanent magnets ring $T_{L P N}$ can be computed from the Eq. (14).

$$
\begin{aligned}
\overline{\overline{\sigma_{\text {magn }}}}(M, t)=\frac{1}{\mu_{0}}\left(\begin{array}{ccc}
B_{r}^{\text {tot }} \cdot B_{\theta}^{\text {tot }}-B_{r}^{\text {tot }}{ }^{2} / 2 & B_{r}^{\text {tot }} \cdot B_{\theta}^{\text {tot }} & 0 \\
B_{r}^{\text {tot }} \cdot B_{\theta}^{\text {tot }} & B_{r}^{\text {tot }} \cdot B_{\theta}^{\text {tot }}-B_{\theta}^{\text {tot }} / 2 & 0 \\
0 & 0 & 0
\end{array}\right)_{\left(\overrightarrow{e_{r}}, \overrightarrow{e_{\theta}}, \overrightarrow{e_{z}}\right)} \\
\\
\left\{\begin{array}{c}
T_{L P N}(t)=R \cdot F_{t}(t) \\
F_{\text {tang }}(t)=\frac{L_{z}}{\mu_{0}} \int_{0}^{2 \pi} B_{r}^{\text {tot }}(t) \cdot B_{\theta}^{t o t}(t) R d \theta
\end{array}\right.
\end{aligned}
$$

The magnetic power transmission can be performed with two conditions: the part of the magnetic energy stored in airgaps which generates magnetic torque defined in Eq. (15) must be different to zero, the average value of the magnetomechanical torque defined in Eq. (16) must also be different to zero.

$$
\begin{gathered}
W_{m L P N}(t)=\frac{e \cdot L_{z}}{\mu_{0}} \int_{0}^{2 \pi} B_{r}^{t o t}(t) \cdot B_{\theta}^{t o t}(t) R d \theta \\
T_{L P N}(t)=-\frac{\partial W_{m L P N}(t)}{\partial \theta_{L P N}}
\end{gathered}
$$

Considering the global induction defined in Eq. (12), the energy stored in airgaps is different to zero only if the condition defined in Eq. (17) is respected. There is then a ratio between the average magnetic torques which corresponds to the gear ratio of the system. Like mechanical epicyclic gears, it is possible to define the ratio (18) where $n_{\text {magn }}$ corresponds to the number of rings external to the pole pieces ring. The Willis equation for a magnetic gear is given in Eq. (19). Then the gear ratio $G_{\text {magn }}$ depends on the fixed ring as shown in Eq. (20) [10]. In these equations, the number of pole pairs and pole pieces corresponds to the number of teeth of the mechanical epicyclic gear.

$$
\begin{gathered}
p_{H P N}+p_{L P N}=Q \\
\lambda_{\text {magn }}=\frac{\omega_{L P N / 0}-\omega_{Q / 0}}{\omega_{H P N / 0}-\omega_{Q / 0}}=\frac{p_{H P N}}{p_{L P N}}(-1)^{n_{\text {magn }}}
\end{gathered}
$$




$$
\begin{gathered}
\omega_{L P N / 0}-\lambda_{\text {magn }} \cdot \omega_{H P N / 0}+\left(\lambda_{\text {magn }}-1\right) \cdot \omega_{Q / 0}=0 \\
\left\{\begin{array}{c}
\omega_{L P N / 0}=0 \rightarrow G_{\text {magn }}=\frac{\omega_{H P N / 0}}{\omega_{Q / 0}}=\frac{\left(\lambda_{\text {magn }}-1\right)}{\lambda_{\text {magn }}} \\
\omega_{Q / 0}=0 \rightarrow G_{\text {magn }}=\frac{\omega_{L P N / 0}}{\omega_{H P N / 0}}=\lambda_{\text {magn }} \\
\omega_{H P N / 0}=0 \rightarrow G_{\text {magn }}=\frac{\omega_{L P N / 0}}{\omega_{Q / 0}}=-\left(\lambda_{\text {magn }}-1\right)
\end{array}\right.
\end{gathered}
$$

\section{Comparison between mechanical and magnetic gears}

\subsection{Transposition of mechanical epicyclic gear arrangements}

To obtain a magnetic interaction between permanent magnets rings, it is not necessary to have the three concentric rings of the magnetic gear. Indeed, like the mechanical epicyclic gears presented Fig. 2, it is possible to have different arrangements with two internal permanent magnet rings or with two external permanent magnet rings as shown in Fig. 5. The arrangement with two internal rings of permanent magnets has been studying by [11],[12] and [13], unlike the arrangements shown in Fig. 5b and Fig. 5c which have not been studied for the moment. For the magnetic gear arrangements shown in Fig. 5b, Fig. 5c and Fig. 5d, the coupling between permanent magnet rings force to have a transverse flux in pole pieces.
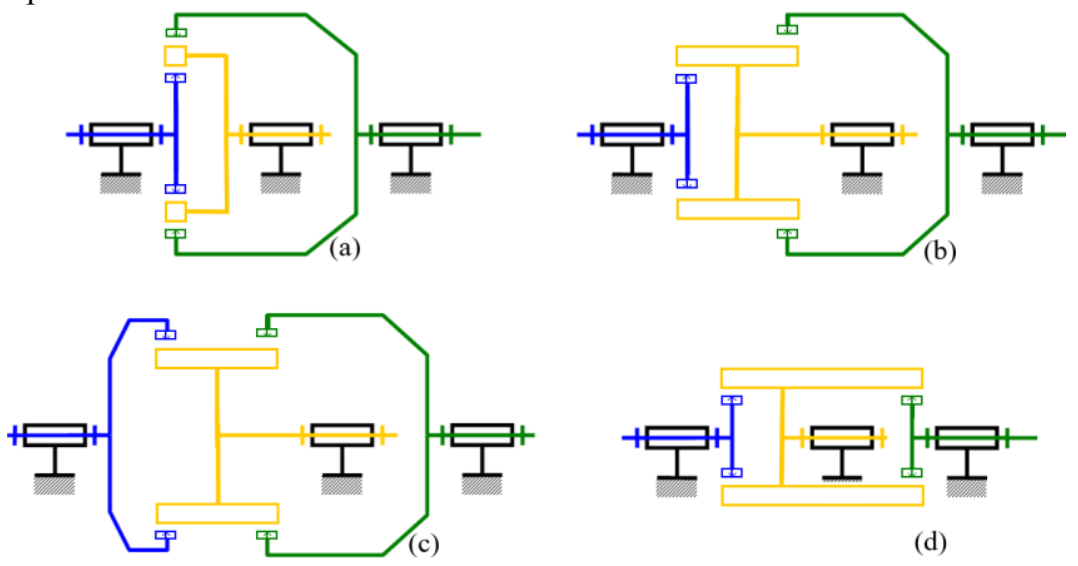

(d)

Fig. 5 Magnetic gear arrangements with (a) a concentric internal and external permanent magnets rings with a pole pieces ring, (b) a non-concentric internal and external permanent magnets ring with a pole pieces ring, (c) two external permanent magnets rings and a pole pieces ring and (d) two internal permanent magnets rings and a pole pieces ring. 


\subsection{Kinematics}

From Eq. (8) and Eq. (20), it is possible to compare in Table 1 the gear ratio of the mechanical and magnetic gears depending on the fixed ring and the arrangement. Table 1 shows that the kinematic behaviour in the same for the mechanical and magnetic gears for the different arrangements and depending on the fixed ring.

Table 1. Gear ratio comparison depending on the arrangement and the fixed ring

\begin{tabular}{|c|c|c|c|}
\hline \multirow[b]{2}{*}{ Config. } & \multicolumn{3}{|c|}{ Fixed rings (mechanical and magnetic) } \\
\hline & $\omega_{H P N / 0}=\omega_{H T N / 0}=0$ & $\omega_{L P N / 0}=\omega_{L T N / 0}=0$ & $\omega_{Q / 0}=\omega_{P C / 0}=0$ \\
\hline $\begin{array}{l}\text { Arrangement } \\
\text { Fig. } 2 \mathrm{a} \text { and Fig. } 5 \mathrm{a}\end{array}$ & $\begin{aligned} G_{\text {magn }} & =\frac{Q}{p_{L P N}} \\
G_{\text {mech }} & =\frac{Z_{N 1}}{Z_{L T N}}\end{aligned}$ & $\begin{aligned} G_{\text {magn }} & =\frac{Q}{p_{H P N}} \\
G_{\text {mech }} & =\frac{Z_{N 2}}{Z_{H T N}}\end{aligned}$ & $\begin{aligned} G_{m a g n} & =-\frac{p_{H P N}}{p_{L P N}} \\
G_{m e c h} & =-\frac{Z_{H T N}}{Z_{L T N}}\end{aligned}$ \\
\hline $\begin{array}{l}\text { Arrangement } \\
\text { Fig. } 2 \mathrm{~b} \text { and Fig. } 5 \mathrm{~b}\end{array}$ & $\begin{aligned} G_{\text {magn }} & =\frac{Q}{p_{L P N}} \\
G_{\text {mech }} & =\frac{Z_{N 1}}{Z_{L T N}}\end{aligned}$ & $\begin{array}{l}G_{\text {magn }}=\frac{Q}{p_{H P N}} \\
G_{\text {mech }}=\frac{Z_{N 2}}{Z_{H T N}}\end{array}$ & $\begin{array}{c}G_{\text {magn }}=-\frac{p_{H P N}}{p_{L P N}} \\
G_{m e c h}=-\frac{Z_{H T N} \cdot Z_{N 1}}{Z_{N 2} \cdot Z_{L T N}}\end{array}$ \\
\hline $\begin{array}{l}\text { Arrangement } \\
\text { Fig. 2c and Fig. 5c }\end{array}$ & $\begin{aligned} G_{\text {magn }} & =\frac{Q}{p_{L P N}} \\
G_{\text {mech }} & =\frac{Z_{N 1}}{Z_{L T N}}\end{aligned}$ & $\begin{aligned} G_{\text {magn }} & =\frac{Q}{p_{H P N}} \\
G_{\text {mech }} & =\frac{Z_{N 2}}{Z_{H T N}}\end{aligned}$ & $\begin{array}{c}G_{\text {magn }}=\frac{p_{H P N}}{p_{L P N}} \\
G_{m e c h}=\frac{Z_{H T N} \cdot Z_{N 1}}{Z_{N 2} \cdot Z_{L T N}}\end{array}$ \\
\hline $\begin{array}{l}\text { Arrangement } \\
\text { Fig. } 2 \mathrm{~d} \text { and Fig. } 5 \mathrm{~d}\end{array}$ & $\begin{aligned} G_{\text {magn }} & =\frac{Q}{p_{L P N}} \\
G_{\text {mech }} & =\frac{Z_{N 1}}{Z_{L T N}}\end{aligned}$ & $\begin{aligned} G_{\text {magn }} & =\frac{Q}{p_{H P N}} \\
G_{\text {mech }} & =\frac{Z_{N 2}}{Z_{H T N}}\end{aligned}$ & $\begin{array}{c}G_{\text {magn }}=\frac{p_{H P N}}{p_{L P N}} \\
G_{m e c h}=\frac{Z_{H T N} \cdot Z_{N 1}}{Z_{N 2} \cdot Z_{L T N}}\end{array}$ \\
\hline
\end{tabular}

\subsection{Load distribution evolution}

Due to the non-contact power transmission between the different rings of the magnetic gear, contrary to the mechanical epicyclic gear, the permanent magnets that correspond to the teeth of the mechanical gear can transmit continuously energy. A comparison can be made between the power transmitted by a tooth of a mechanical gear and the power transmitted by a magnet of a magnetic gear. For this, we consider that the fixed ring is the planetary carrier for the epicyclic gear and the pole pieces ring for the magnetic gear. An example is given in Fig. 6 where the power transmission is performed for the mechanical epicyclic gear configuration show in Fig. $3\left(Z_{L T N}=20, Z_{H T N}=40, Z_{N 1}=Z_{N 2}=10\right.$ and $\left.N=4\right)$ and for the magnetic gear configuration show in Fig.4 $\left(p_{L P N}=2, p_{H P N}=5\right.$ and $\left.Q=7\right)$. 


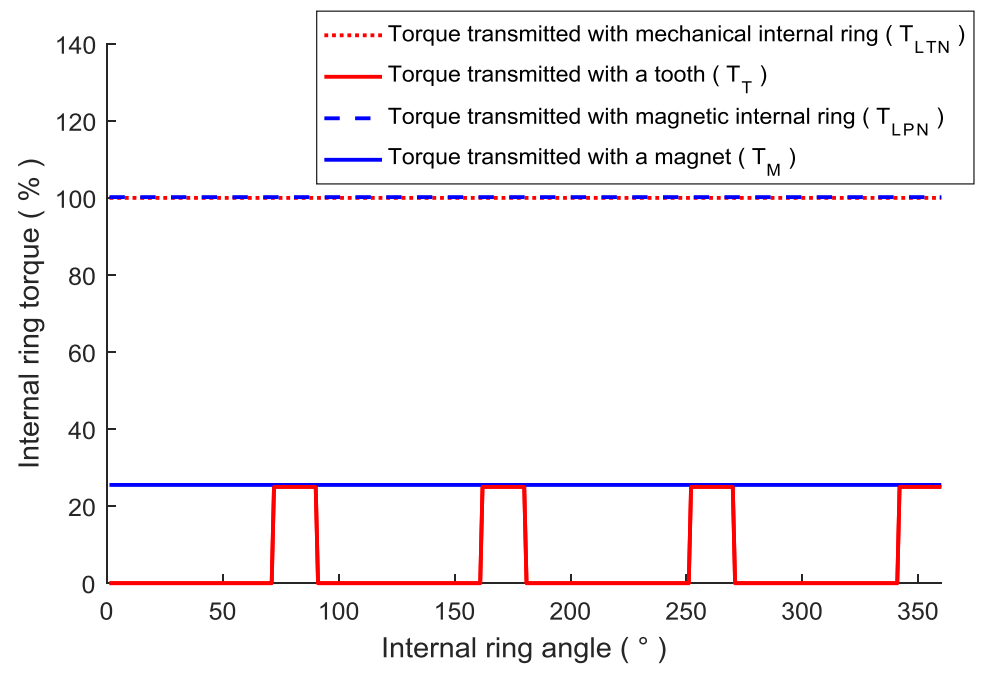

Fig. 6 Load repartition evolution in function of the internal ring angle for a mechanical gear with $Z_{L T N}=20, Z_{H T N}=40, Z_{N 1}=Z_{N 2}=10$ and $N=4$ and a magnetic gear with $p_{L P N}=2, p_{H P N}=5$ and $Q=7$.

Fig. 6 shows that a tooth of a ring of a mechanical epicyclic gear transmits tangential load only when the tooth is in contact with a corresponding tooth of a satellite. On the opposite, the magnets of the magnetic gear transmit tangential load at every moment since there is a non-contact power transmission. It is then possible to express the percentage of the torque transmitted by a tooth $T_{T}$ or a magnet $T_{M}$ according to the gear type defined in Eq. (21) where $T_{L T N}$ and $T_{L P N}$ are defined in Eq. (3) and Eq. (16). It is also possible to express the utilization rate of a tooth $\tau_{T}$ or a magnet $\tau_{M}$ according to the studied gear type defined in Eq. (22).

$$
\begin{gathered}
\left\{\begin{array}{c}
T_{T}(\%)=\frac{100}{N} \\
T_{M}(\%)=\frac{100}{2 \cdot p_{L P N}}
\end{array}\right. \\
\left\{\begin{array}{c}
\tau_{T}(\%)=\frac{100 \cdot N}{Z_{L T N}} \\
\tau_{M}(\%)=100
\end{array}\right.
\end{gathered}
$$

This result contributes to demonstrate that the power density of magnetic gear is higher than mechanical epicyclic gear power density [5]. The benefit of a magnetic gear over mechanical gearboxes is greater for high-torque application since the number of teeth or pole increases more quickly than the number of satellites. However, the permanent magnet costs slow down the development of magnetic gear industrial applications. 


\section{Conclusions}

This article presents a comparison between magnetic gears with modulating ring and mechanical epicyclic gears. It illustrates a kinematic analogy between the two gears for different arrangements. On the other hand, this study shows that the load repartition evolution is fundamentally different since a tooth of a mechanical gear transmits the power intermittently while a magnet of a magnetic gear transmits the power continuously. Magnetic gears have a high potential for applications requiring high reliability and a high power and torque density such as offshore wind turbines.

\section{References}

1. J. Keller, Y. Guo, and L. Sethuraman, "Gearbox Reliability Collaborative Investigation of Gearbox Motion and High-Speed-Shaft Loads," NREL, Tech. Rep. TP-5000-65321, 2016.

2. W. Teng, X. Ding, X. Zhang, Y. Liu, and Z. Ma, "Multi-fault detection and failure analysis of wind turbine gearbox using complex wavelet transform," Renew. Energy, vol. 93, pp. 591-598, 2016.

3. K. Atallah and D. Howe, "A novel high-performance magnetic gear," IEEE Trans. Magn., vol. 37, no. 4, pp. 2844-2846, 2001.

4. T. B. Martin, "Magnetic transmission,” Pat. US3378710, 1968.

5. E. Gouda, S. Mezani, L. Baghli, and A. Rezzoug, "Comparative study between mechanical and magnetic planetary gears," IEEE Trans. Magn., vol. 47, no. 2, pp. 439-450, 2011.

6. D. Matt, J. Jac, and N. Ziegler, "Design of a Mean Power Wind Conversion Chain with a Magnetic Speed Multiplier," in Chap. 10 of InTech book "Advances in Wind Power, 2012, pp. 247-266.

7. A. Penzkofer and K. Atallah, "Magnetic Gears for High Torque Applications," IEEE Trans. Magn., vol. 50, no. 11, 2014.

8. J. J. Coy, D. P. Townsend, and E. V Zaretsky, “Gearing,” 1985.

9. R. Mathis and Y. Remond, "Kinematic and dynamic simulation of epicyclic gear trains," Mech. Mach. Theory, vol. 44, no. 2, pp. 412-424, 2009.

10. M. Desvaux, B. Traullé, R. L. G. Latimier, S. Sire, B. Multon, and H. Ben Ahmed, "Computation Time Analysis of the Magnetic Gear Analytical Model," IEEE Trans. Magn., vol. 53, no. 5, pp. 1-10, 2017.

11. L. Y. L. Yong, X. J. X. Jingwei, P. K. P. Kerong, and L. Y. L. Yongping, "Principle and simulation analysis of a novel structure magnetic gear," Int. Conf. Electr. Mach. Syst., no. 1, pp. 3845-3849, 2008.

12. Y. Li, J. W. Xing, Y. P. Lu, and Z. J. Yin, "Torque analysis of a novel non-contact permanent variable transmission," IEEE Trans. Magn., vol. 47, no. 10, pp. 4465-4468, 2011.

13. W. Bomela, J. Z. Bird, and V. M. Acharya, "The performance of a transverse flux magnetic gear," IEEE Trans. Magn., vol. 50, no. 1, pp. 8-11, 2014. 